\title{
Prüfung der Zeitdilatation mit Hilfe des Mößbauer-Effektes
}

\author{
H. HöNL und F. BENNEwitz \\ Institut für theoretische Physik der Universität Freiburg i. Br. \\ (Z. Naturforschg. 21 a, 867-869 [1966] ; eingegangen am 7. März 1966) \\ Professor Dr. W. Gentner zum 60. Geburtstag gewidmet
}

\begin{abstract}
A Lorenz-invariant treatment of the second order Doppler effect in rotational motions is given. The calculation is then performed in a generally covariant manner. Experimental consequences concerning the mechanical stability of clocks are discussed, and attention is drawn to a possible refinement of the rotational experiments.
\end{abstract}

Während in früheren Experimenten der relativistische Doppler-Effekt nur an Teilchen mit hohen Geschwindigkeiten (z.B. Kanalstrahlen ${ }^{*}$ ) nachgeprüft werden konnte, gestattet die hohe Meßgenauigkeit des Mössbauer-Effektes das Registrieren selbst des quadratischen DopplER-Effektes bei thermischen Geschwindigkeiten von etwa $100 \mathrm{~m} / \mathrm{sec}$. Damit konnten in einer Reihe von Experimenten ${ }^{1-5}$ die Grundlagen der speziellen Relativitätstheorie neu überprüft werden. Als den Doppler-Effekt hervorrufende Geschwindigkeit wurde sowohl die thermische Bewegung ${ }^{5}$ als auch (in den übrigen Experimenten) die Bewegung von Absorber und Emitter auf einem Rotor verwandt.

Die Temperaturbewegung der Kerne im Gitter bewirkt nicht nur eine Verbreiterung der MössbautrLinie, sondern auch eine Verschiebung ihres Maximums (und als Effekt höherer Ordnung eine Veränderung der Linienform). Die Temperaturabhängigkeit der Lage des Maximums ist somit ebenfalls ein $\mathrm{Ma}$ für den Doppler-Effekt. In Überlegungen von WEGENER ${ }^{6}$ wird angenommen, daß der Kern wie ein klassischer Oszillator sendet. Da die Sendezeit (Lebensdauer des angeregten Zustandes) wesentlich größer ist als die Perioden der Gitterschwingungen,

* H. Otting, Phys. Z. 40, 681 [1939].

1 H. J. Hay, J. P. Schiffer, T. E. Cranshaw u. P. A. EgelStafF, Phys. Rev. Letters 4, 165 [1960].

2 D. C. Champeney, G. R. Isaak u. A. M. Khan, Nature London 198, 1186 [1963]; Phys. Letters 7, 241 [1963]; Proc. Phys. Soc. London 85, 583 [1965].

3 W. Kündig, Phys. Rev. 129, 2371 [1962].

${ }^{4}$ K. C. Turner u. H. A. Hill, Phys. Rev. 134, B 252 [1964].

5 R. V. Pound u. G. A. Rebra, Phys. Rev. Letters 4, 274 [1960].

6 H. WEGEner, Der Mößbauereffekt, Bibliographisches Institut Mannheim 1965, p. 103.

7 Eine exaktere halbklassische Rechnung müßte berücksichtigen, daß das Quant momentan emittiert und absorbiert wird; man muß also die Gleichung für die gegenseitige wird die Kernbewegung über die Gitterschwingungen gemittelt und die Zeitdilatation wird nur von $\overline{v^{2}}$ (mittleres Geschwindigkeitsquadrat) abhängig, d. h. nur von der Temperatur, unabhängig von der speziellen Struktur des Gitters. Man erhält als relative Temperaturverschiebung pro Grad Kelvin ${ }^{7}$

$$
\frac{\Delta v(T)}{v_{0}}=\frac{E(T)}{2 N M c^{2}}
$$

( $M$ Atomgewicht, $E$ Energie pro Mol, $N$ Loschmidтsche Zahl, $c$ Lichtgeschwindigkeit).

Die für die Temperaturverschiebung gemessenen Werte $^{5}$ stimmen mit der Berechnung innerhalb des Meßfehlers überein. Der Vergleich ist jedoch nicht sehr überzeugend wegen der noch hohen Meßungenauigkeit und der Unsicherheit infolge der verwendeten Vereinfachungen.

\section{Speziell-relativistische Theorie}

Eine Bestätigung der Zeitdilatation mit größerer Genauigkeit ist jedoch möglich bei dem Frequenzvergleich zwischen Emitter und Absorber gleicher Temperatur auf der rotierenden Scheibe. Bei kovarianter Rechnung lassen wir beliebige (feste)

Doppler-Verschiebung zweier Kerne nacheinander über die Geschwindigkeitsverteilung im Emitter- und Absorbergitter bei den jeweiligen Temperaturen mitteln, die noch vom Phononenspektrum des Kristalls abhängt. - Unter Verwendung der harmonischen Oszillator-Eigenfunktionen ergibt eine quantenmechanische Behandlung ${ }^{8}$ ebenfalls obiges Ergebnis (1), wobei aber nicht die Zeitdilatation, sondern $E=m c^{2}$ verwandt wird. Beide Beziehungen sind jedoch äquivalent, wenn man, wie üblich, die (davon noch unabhängige) Isotropie des 3-dimensionalen Raumes und Konstanz von $c$ in allen Inertialsystemen voraussetzt (A. Robertson, Rev. Mod. Phys. 21, 378 [1949ך).

8 B. D. Josephson, Phys. Rev. Letters 4, 341 [1960]. 
Lagen von Emitter E und Absorber A zu, die mit gleicher Winkelgeschwindigkeit $\omega$ um die gemeinsame Rotationsachse 0 rotieren; $r, \Theta, \varphi$ seien Zylinderkoordinaten $(\Theta=0$ kennzeichnet die Ebene durch A senkrecht zur Rotationsachse). Die Winkel, Geschwindigkeiten und Radiusvektoren beziehen sich auf das Laborsystem. Da wir im folgenden ausschließlich die Vierer-Vektoren $u^{\mu}, k^{\mu}$ und die Skalare $v$ benutzen, ist die weiterhin durchzuführende Rechnung LORENTZ-invariant.

Die Radiusvektoren $\boldsymbol{r}_{\mathrm{E}}$ des Emitters zur Emissionszeit und $\boldsymbol{r}_{\mathrm{A}}$ des Absorbers zur Absorptionszeit mögen den Winkel $\varphi$ einschließen. Die Rotationsachse sei die $x^{3}$-Achse, als $x^{2}$-Achse wählen wir $\boldsymbol{r}_{\mathrm{A}}$ im Augenblick der Absorption. Als Bezugssystem wählen wir also das Inertialsystem, gegen welches die Scheibe rotiert. Für die Vierergeschwindigkeit des Emitters zur Zeit der Emission $u_{\mathrm{E}}^{\mu}=\frac{\mathrm{d} x_{\mathrm{E}}^{\mu}}{c \mathrm{~d} \tau_{\mathrm{E}}}(\tau$ MrNкоwsкг-Eigenzeit, die Mınкоwsкi-Metrik habe die Signatur -2 ) erhalten wir dann

$$
u_{\mathrm{E}}^{\mu}=\frac{1}{\sqrt{1-\beta_{\mathrm{E}}^{2}}}\left(-\beta_{\mathrm{E}} \cos \varphi, \beta_{\mathrm{E}} \sin \varphi, 0,1\right)
$$

und für die Vierergeschwindigkeit des Absorbers zur Absorptionszeit

$$
u_{\mathrm{A}}^{\mu}=\frac{1}{\sqrt{1-\beta_{\mathrm{A}}^{2}}}\left(-\beta_{\mathrm{A}}, 0,0,1\right) .
$$

Der Wellenvektor des Photons sei

$k^{\mu}=v(-\sin \alpha \cos \Theta, \cos \alpha \cos \Theta, \sin \Theta, 1)$.

Dabei sind $\Theta$ und $\alpha$ die sphärischen Winkel des 3-dimensionalen Wellenvektors, $v$ ist die Frequenz der Welle im Laborsystem. Für die Emitter- und Absorberfrequenzen (Eigenfrequenzen des Emitters $v_{\mathrm{E}}$ und Frequenz der Welle $\nu_{\mathrm{A}}$ im Absorbersystem) gilt $v_{\mathrm{E}}=k_{u} u_{\mathrm{E}}^{\mu}$ und $v_{\mathrm{A}}=k_{\mu} u_{\mathrm{A}}^{\mu}$. Hiermit erhält man aus $(2 \mathrm{a}, \mathrm{b})$ und $(3)$

$$
v_{\mathrm{A}}=\nu_{\mathrm{E}} \frac{1-\beta_{\mathrm{A}} \sin \alpha \cos \Theta}{1-\beta_{\mathrm{E}} \sin (\alpha+\varphi) \cos \Theta} \frac{\sqrt{1-\beta_{\mathrm{E}}{ }^{2}}}{\sqrt{1-\beta_{\mathrm{A}}}}
$$

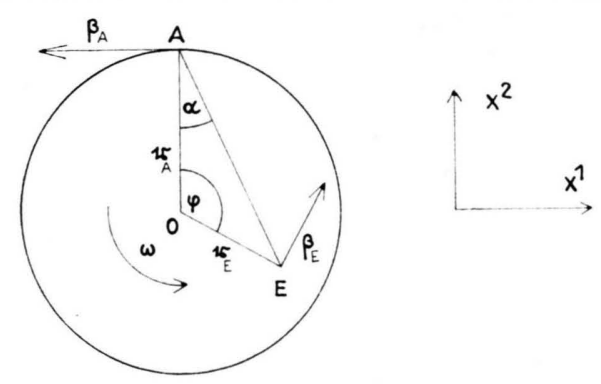

Fig. 1. Parallelprojektion längs der Rotationsachse 0 . der winkelabhängige Faktor fällt hierbei wegen $\beta_{\mathrm{A}} \sin \alpha=\beta_{\mathrm{E}} \sin (\alpha+\varphi)$ (siehe Abb. 1) heraus und wir erhalten das einfache Resultat:

$$
\nu_{\mathrm{A}}=\nu_{\mathrm{E}}\left(\sqrt{1-\beta_{\mathrm{E}}^{2}}\right) /\left(\sqrt{1-\beta_{\mathrm{A}}{ }^{2}}\right) .
$$

(Durch Isomerieverschiebung konnte auch das in früheren Versuchen offen gebliebene Vorzeichen von $\Delta v$ experimentell bestimmt werden ${ }^{2}, \operatorname{vgl.}{ }^{3}$.)

\section{Allgemein kovariante Fassung der Theorie}

Die voranstehende Rechnung ist ersichtlich LoREntzinvariant. Wir wollen sie andererseits auch allgemein kovariant durchführen, indem wir zu dem sich mitdrehenden Koordinatensystem übergehen und dabei die Invarianz des metrischen Feldes gegen eine spezielle Bewegungsgruppe benutzen. Im mitrotierenden Bezugssystem $(r, \psi, z, c t)$ gilt eine Riemanssche Geometrie. Gemäß der Transformation vom Laborins rotierende System

$x^{1}=r \cos (\omega t+\psi), x^{2}=r \sin (\omega t+\psi), x^{3}=z, x^{4}=c t$

wird der metrische Tensor $g_{\mu \nu}$ :

$$
\begin{gathered}
g_{11}=-1, g_{33}=-1, g_{22}=-r^{2}, g_{44}=1-\frac{\omega^{2}}{c^{2}} r^{2}, \\
g_{24}=g_{42}=-\frac{\omega}{c} r^{2},
\end{gathered}
$$

alle anderen Komponenten verschwinden. Es seien nun $s_{\mathrm{A}}, s_{\mathrm{E}}$ die Riemansschen Bogenlängen auf den Weltlinien des Absorbers und Emitters, $t_{\mathrm{A}}, t_{\mathrm{E}}$ die entsprechenden Koordinatenzeiten. Der Emitter sendet in der Eigenzeit $\mathrm{d} s_{\mathrm{E}}$ genau $n$ Wellenzüge der Eigen-Frequenz $\nu_{\mathrm{E}}$ aus, die der Absorber während der Eigenzeit $\mathrm{d} s_{\mathrm{A}}$ als Frequenz $v_{\mathrm{A}}$ empfängt. Da $n$ als ganze Zahl eine Invariante ist, gilt

$$
\nu_{\mathrm{E}} \mathrm{d} s_{\mathrm{E}}=\boldsymbol{v}_{\mathrm{A}} \mathrm{d} s_{\mathrm{A}}=n .
$$

Das Feld auf der rotierenden Scheibe ist ein stationäres Feld, es läßt also eine Bewegungsgruppe längs der $t$-Achse zu. Deshalb schneiden zwei längs einer $t$-Achse verschobene Lichtkegel auf allen $t$-Achsen gleiche Stücke aus: $\mathrm{d} t_{\mathrm{A}}=\mathrm{d} t_{\mathrm{E}}$. Die Weltlinien von Absorber und Emitter sind $t$-Achsen, so daß mit

folgt:

$$
\mathrm{d} s^{2}=g_{\mu \nu} \mathrm{d} \xi^{\mu} \mathrm{d} \xi^{\nu},\left(\xi^{\mu}\right)=(r, \psi, z, c t)
$$

$$
\begin{aligned}
& \mathrm{d} r=\mathrm{d} \psi=\mathrm{d} z=0, \mathrm{~d} s_{\mathrm{E}}=\sqrt{\left(g_{44}\right)_{\mathrm{E}}} \mathrm{d} t_{\mathrm{E}}, \\
& \mathrm{d} s_{\mathrm{A}}=\sqrt{\left(g_{44}\right)_{\mathrm{A}}} \mathrm{d} t_{\mathrm{A}},
\end{aligned}
$$

damit wird

$$
v_{\mathrm{A}}=v_{\mathrm{E}} \frac{\mathrm{d} s_{\mathrm{E}}}{\mathrm{d} s_{\Lambda}}=v_{\mathrm{E}} \sqrt{\left(g_{44}\right)_{\mathrm{E}}} / \sqrt{\left(g_{44}\right)_{\mathrm{A}}} .
$$


Mit dem Ausdruck für $g_{44}$ erhalten wir wieder das obige Ergebnis (4)

$$
v_{\mathrm{A}}=\nu_{\mathrm{E}}\left(\sqrt{1-\beta_{\mathrm{E}}^{2}}\right) /\left(\sqrt{1-\beta_{\mathrm{A}}^{2}}\right) .
$$

Da der zugrunde liegende Raum global „MiNkowsKısch" ist (alle Krümmungskomponenten verschwinden), brauchten wir zur Lösung des Problems nicht die Einsternschen Feldgleichungen zu verwenden oder auch nur die Geodätengleichung zu lösen. Die allgemein-relativistische Behandlung ist hier ersichtlich eine kovariante Form der speziell-relativistischen Rechnung. Deshalb erlaubt das Ergebnis auch keine Schlüsse hinsichtlich der Gültigkeit der allgemeinen Relativitätstheorie bzw. des Äquivalenzprinzips (vgl. dagegen ${ }^{9,10}$ ). Dies ist schon daraus ersichtlich, daß man den Effekt allein aufgrund der speziellen Relativitätstheorie erklären kann, in welche das Äquivalenzprinzip gar nicht eingeht. Die Tatsache, daß die Ruhsysteme von Emitter und Absorber beschleunigt sind (in den bisher durchgeführten Experimenten nur dasjenige des Absorbers), soll hierbei keine Rolle spielen (vgl. jedoch den Schlußabsatz 3); die Frequenzverschiebung ist dann nur von den Geschwindigkeiten von Emitter und Absorber abhängig.

\section{Folgerungen für das Experiment}

Man wird sich jedoch fragen müssen, ob überhaupt Beschleunigungen den Gang von Uhren beeinflussen können. Dabei ist zweierlei zu überlegen:

1. Welche Gangänderung ergibt die allgemeine Relativitätstheorie?

2. Bleibt die Uhr bei Beschleunigungen mechanisch heil?

Wie für den Spezialfall von Rotationsbewegungen gezeigt wurde, ergibt die allgemeine Relativitätstheorie in ebenen Raum-Zeit-Gebieten Unabhängigkeit des Ganges „idealer Uhren“ von Beschleunigun-

9 M. A. Tonnelat, Les Vérifications Expérimentales de la Relativité Générale, Masson et Cie., p. 165 (Paris 1964). gen. Falls die Beschleunigung jedoch durch andere als Gravitationswechselwirkung hervorgerufen wird, wird hierbei im allgemeinen vorausgesetzt, daß diese den Gang der Uhr nicht beeinflussen sollen. Da wir nun andererseits die Gültigkeit der speziellen Relativitätstheorie voraussetzen, so kann durch obige Versuche die Gültigkeit dieses Postulats geprüft werden ${ }^{11}$. Das Ergebnis der Rotorexperimente ist dann, daß bei (konstant gerichteten) infolge der CoulombKräfte aufretenden Beschleunigungen von $10^{5} \mathrm{~g}$, die den thermischen Beschleunigungen überlagert sind, der $\mathrm{Fe}^{57}$-Kern als Uhr eine Ganggenauigkeit von mindestens $5 \cdot 10^{-15}$ besitzt (die relative Energieänderung $\Delta E / E$ des ersten angeregten Niveaus ist kleiner als der relative Meßfehler des Maximums der $E$-Verteilung von etwa $5 \cdot 10^{-15}$ ).

Den Effekt der thermischen Beschleunigungen von etwa $10^{16} \mathrm{~g}$ kann man dagegen nicht über den Meßfehler der Temperaturverschiebung abschätzen, da die Beschleunigungen isotrop sind; ohne eine eingehende Theorie der Uhr (Deformation des Kerns) läßt sich dieser nur durch die Doppler-Verbreitung der Linie begrenzen. Damit erhält man, daß CouLомв-Beschleunigungen von $10^{16} \mathrm{~g}$ im $\mathrm{Fe}^{57}$-Kern eine relative Gangdifferenz (relative $E$-Niveau-Verschiebung $\Delta E / E$ ) von weniger als $10^{-12}$ verursachen.

Bei der speziell-relativistischen Behandlung der Rotorexperimente mußten wir Winkel einführen, die die gegenseitige Lage von Emitter und Absorber bestimmen. Die sich ergebende Frequenzverschiebung war jedoch nur noch von den radialen Abständen von der Rotationsachse abhängig, so daß man Emitter und Absorber zu konzentrischen Zylindern ausdehnen kann. Damit fielen nicht nur elektronische Tore fort, sondern die wesentlich größeren Zählraten verringerten vor allem auch den statistischen Fehler. Wir möchten auf diese Möglichkeit der Verbesserung der experimentellen Anordnung ausdrücklich hinweisen.

10 T. E. Cranshaw, in Proc. Int. School of Phys. E. Fermi, course 20: Evidence for Gravitational Theories, p. 208 [1961].

C. W. Sherwin, Phys. Rev. 120, 17 [1960]. 